Research Paper

\title{
Plasmid-mediated quinolone resistance (PMQR) and mutations in the topoisomerase genes of Salmonella enterica strains from Brazil
}

\author{
Rafaela Ferrari ${ }^{1}$, Antonio Galiana ${ }^{2}$, Rosa Cremades $^{2}$, Juan Carlos Rodríguez ${ }^{2}$, \\ Marciane Magnani ${ }^{3}$, M.C.B. Tognim ${ }^{4}$, Tereza C.R.M. Oliveira ${ }^{1}$, Gloria Royo ${ }^{2}$ \\ ${ }^{1}$ Departamento de Ciência e Tecnologia de Alimentos, Centro de Ciências Agrárias, \\ Universidade Estadual de Londrina, Londrina, PR, Brazil. \\ ${ }^{2}$ Servicio de Microbiología, Hospital General Universitario de Elche, \\ Universidad Miguel Hernández, Elche, Spain. \\ ${ }^{3}$ Departamento de Engenharia de Alimentos, Centro de Tecnologia, Universidade Federal da Paraíba, \\ São João, PB, Brazil. \\ ${ }^{4}$ Departamento de Análises Clínicas, Centro de Ciências da Saúde, Universidade Estadual de Maringá, \\ Maringá, PR, Brazil.
}

Submitted: December 16; Approved: July 23, 2012.

\begin{abstract}
The objective of this study was to identify mutations in the Quinolone Resistance Determining sources Regions (QRDR) of the gyrA, gyrB, parC, and parE genes and to determine if any of the qnr variants or the $a a c\left(6^{\prime}\right)-I b-c r$ variant were present in strains of Salmonella spp. isolated in Brazil. A total of 126 Salmonella spp. strains from epidemic $(\mathrm{n}=114)$ and poultry $(\mathrm{n}=12)$ origin were evaluated. One hundred and twelve strains (88.8\%) were resistant to nalidixic acid (NAL) and 29 (23.01\%) showed a reduced susceptibility to ciprofloxacin (Cip). The mutations identified were substitutions limited to the QRDR of the gyrA gene in the codons for Serine 83, Aspartate 87 and Alanine 131. The sensitivity to NAL seems to be a good phenotypic indication of distinguishing mutated and nonmutated strains in the QRDR, however the double mutation in gyrA did not cause resistance to ciprofloxacin. The qnrA1 and qnrB19 genes were detected, respectively, in one epidemic strain of S. Enteritidis and one strain of S. Corvallis of poultry origin. Despite previous detection of $q n r$ genes in Brazil, this is the first report of qnr gene detection in Salmonella, and also the first detection of qnrB19 gene in this country. The results alert for the continuous monitoring of quinolone resistance determinants in order to minimize the emergence and selection of Salmonella spp. strains showing reduced susceptibility or resistance to quinolones.
\end{abstract}

Key words: Salmonella, PMQR, QRDR mutation, quinolone, ciprofloxacin.

\section{Introduction}

Salmonellosis is a common cause of foodborne diseases and is a serious public health problem (Arkin, 2008). Fluoroquinolones (FQs) are used to treat invasive salmonellosis in humans and animals, but in recent years, studies have reported an increase in the number of clinical isolates resistant or with a reduced susceptibility to these compounds (Ercis et al., 2006; Kownhar et al., 2007; Souza et al., 2010).
The FQs inhibit the activity of the enzymes topoisomerase II (DNA-gyrase) and topoisomerase IV, inducing cell death. Mutations in the quinolone resistance-determining regions (QRDRs) of the gyrA and $\operatorname{parC}$ genes alter the DNA-gyrase binding sites of these antibiotics and can result in resistance to quinolones. In Salmonella spp., these mutations are related to nalidixic acid (NAL) resistance and reduced susceptibility to FQs, such as ciprofloxacin (Cip) (Cavaco and Aarestrup, 2009; Hopkins et al., 2005). Other mechanisms associated with low susceptibility or resis-

Send correspondence to R. Ferrari. Centro de Ciências Agrárias, Departamento de Ciência e Tecnologia de Alimentos, Universidade Estadual de Londrina, Av. Celso Garcia Cid s/n, Londrina, PR, Brazil. E-mail: rafaelaferrari@yahoo.com.br. 
tance to FQs, such as, efflux pump over-expression, modification of porin regulation, and plasmid-mediated quinolone resistance (PMQR) have been reported in Salmonella (Sjolund-Karlsson et al., 2010).

The PMQR qnrA gene encodes a 218-amino acid protein, a member of a family of pentapeptides that protect DNA-gyrase and topoisomerase IV from the quinolones' activity (Martinez-Martinez et al., 1998; Tran et al., 2005). This pentapeptide blocks the action of Cip, resulting in a low-level quinolone resistance with an increase in the minimal inhibitory concentration of ciprofloxacin (CipMIC) (Robicsek et al., 2006). At present, the qnr gene is known to encode six proteins: QnrA, with variants from QnrA1 to QnrA6; QnrB, with variants from QnrB1 to QnrB20; QnrS, with variants from QnrS1 to QnrS3; QnrVC; QnrC; and QnrD (Cavaco and Aarestrup, 2009; Fonseca et al., 2008; Robicsek et al., 2006; Wang et al., 2009). Another PMQRbased resistant mechanism involves the ciprofloxacin resistance ( $c r$ ) variant of the $a a c\left(6^{\prime}\right)-I b$ gene, which encodes an aminoglycoside acetyltransferase. This variant contains substitutions in codons 102 and 179 conferring a reduced susceptibility to Cip by $\mathrm{N}$-acetylation at the amino nitrogen on its piperazinyl substituent (Robicsek et al., 2006).

In the present study, Salmonella spp. strains from epidemic and poultry origins isolated in Brazil were screened for mutations in the QRDRs of the gyrA, gyrB, parC, and parE genes and for the presence of any of the $q n r$ variants and the $a a c\left(6^{\prime}\right)-I b-c r$ variant.

\section{Materials and Methods}

Strains of Salmonella spp. A total of 126 Salmonella spp. strains from epidemic (i.e., isolates from outbreaks) and poultry origins, isolated between 1999 and 2007 in Parana State, Brazil, were evaluated. The strains were obtained from the Central Laboratory of Parana State (LACEN, Curitiba, Parana) and serotyped by the Osvaldo Cruz Foundation (Rio de Janeiro, Brazil). The strains were kept at $-15{ }^{\circ} \mathrm{C}$ in brain-heart infusion broth (BHI) (Difco ${ }^{\circledR}$ ) containing $15 \%$ glycerol. The resistance of these strains to NAL, by disc diffusion and, the CipMIC were previously determined by Souza et al. (2010). Among all of the strains, $112(88.8 \%)$ were resistant to NAL, and $29(23.01 \%)$ showed a reduced susceptibility to Cip with a CipMIC from $0.125 \mu \mathrm{g} / \mathrm{mL}$ to $0.5 \mu \mathrm{g} / \mathrm{mL}$ (Souza et al., 2010). Of the tested strains, 114 were of epidemic origin and 12 were from poultry.

Isolation, Amplification and Sequencing of DNA. The strains were cultivated overnight in BHI agar $\left(\right.$ Difco $^{\circledR}$ ) at $37^{\circ} \mathrm{C}$. The DNA was isolated using $10 \%$ Chelex-100. To identify the PMQR genes, including the qnrA, qnrB1, $q n r B 5$, qnrB19, qnrS1, qnrC, and $q n r D$ alleles as well as $a a c\left(6^{\prime}\right)-I b-c r$, the DNA was amplified according the processes described by Gay et al. (2006), Cavaco and Aarestrup (2009) and Wang et al. (2009). The positive genes
qnrA, qnrB1, qnrB5, qnrS1, qnrC, qnrD and aac(6)Ib-cr were used as positive controls. The QRDR of the gyrA, $\operatorname{gyr} B, \operatorname{par} C$, and $\operatorname{par} E$ genes was amplified following the method described by Eaves et al. (2004). The sequence analysis of the PCR products was performed by Macrogen Laboratory (Seoul, Korea).

Sequence analysis. The sequences obtained were compared with those deposited in the database of the National Center for Biotechnology Information (NCBI, Hyperlink http://www.ncbi.nlm.nih.gov/www.ncbi.nlm. nih.gov) using BLAST searches.

\section{Statistic analysis}

The results were analyzed using SPSS Statistics 18.0 software. The geometric means (GMM) of the CipMIC, which indicate the central tendency, were calculated using the formula $\sqrt[n]{y 1 y 2 y 3 \ldots y n}$, where $y$ represents the CipMIC of each individual strain and $n$ is the number of CipMIC used (Ling et al., 2006).

\section{Results and Discussion}

\section{Susceptibility to quinolones and mutations in the topoisomerase genes}

The 14 Salmonella spp. strains (11.1\%) that were NAL-susceptible did not contain mutations in the QRDRs of gyrA, gyrB, parC and parE. Seventy and three strains (57.9\%) from the 112 NAL-resistant strains (88.8\%) analyzed contained mutations in QRDR gyrA gene, with 60 having one and 13 two mutations. No mutations were found in the other genes. These results suggest that NAL susceptibility could indicate QRDR mutation occurrence. According to Cavaco and Aarestrup (2009), the NAL-MIC could clearly differentiate between susceptible strains and strains with one or more QRDR mutations.

Of the 13 NAL-resistant strains with two gyrA mutations, only one, belonging to the Enteritidis serovar, was susceptible to Cip with MIC of $0.064 \mu \mathrm{g} / \mathrm{mL}$. Among the other strains, $11 S$. Enteritidis strains and one $S$. Johannesburg strain showed a reduced susceptibility to this FQ, with MIC $\geq 0.125 \mu \mathrm{g} / \mathrm{mL}$. This reduced susceptibility was also observed in 9 of the 39 NAL-resistant strains without a mutation in the gyrA gene. Similar results were reported by Cavaco and Aarestrup (2009), who also observed a reduced susceptibility or resistance to Cip in strains without $\mathrm{gyrA}$ mutations, probably due to other resistance mechanisms.

The geometric mean (GMM) of the CipMIC for the 73 strains with gyrA mutations was higher than that of the 53 strains without mutations, with means of $0.10 \mu \mathrm{g} / \mathrm{mL}$ and $0.06 \mu \mathrm{g} / \mathrm{mL}$, respectively. Although many of the isolates of Salmonella spp. are considered susceptible to FQs according to CLSI criteria (Aarestrup et al., 2003; Giraud et al., 2006; Gunell et al., 2009; Souza et al., 2010), there have been an increasing number of reports documenting the 
incidence of strains with a reduced susceptibility to these compounds, including cases of therapeutic failure (Aarestrup et al., 2003; Crump et al., 2003; Cui et al., 2009; Piddock, 2002; Ricci and Piddock, 2009; Vashist et al., 2009).

All of the mutations identified in this study were substitutions in the QRDR of the gyrA gene; Serine 83 (Ser 83), Aspartate 87 (Asp 87) or Alanine 131 (Ala 131) codons (Table 1). The Ser 83 mutations were found in 41 strains, with substitutions by Tyrosine (Tyr) and Phenylalanine (Phe) in 19 and 22 strains, respectively. The Asp 87 mutations were found in 44 strains, with substitutions by Asparagine (Asn), Tyr, and Glycine (Gly) in 31, 9, and 4 strains, respectively. The Ala 131 mutation was found in only one strain, with a substitution by Gly. The substitutions observed in the present study in Salmonella spp. strains from epidemic and poultry origins were previously described in animals and humans by Giraud et al. (2006). Although mutations were found in the isolates from epidemic and poultry origins, a direct relationship between the origin of the strain and the type of mutation could not be established.

Both the positions of the mutations and the type of substitution found in the mutated region differed among the serovar type in this study. In the five strains belonging to $S$. Enteritidis, four substitutions were found in Asp $87 \rightarrow$ Gly and one in Ala $131 \rightarrow$ Gly. In addition, the Asp $87 \rightarrow$ Tyr mutation was found in nine strains including seven Enteritidis and two Johannesburg serovars. The Asp $87 \rightarrow$ Asn mutation was present in 31 strains belonging to 5 different serovars. The most common mutations were substitutions of Asp 87 in the serovar Enteritidis, which accounted for $50.8 \%$ of the analyzed strains. This finding is in agreement with the results reported by Soto et al. (2003), who also found a greater frequency of mutations in the codon Asp 87.

Giraud et al. (2006) reported that mutations in Ser 83 and Asp 87 were not equally distributed among the serovars of Salmonella, with a greater frequency of Ser 83 mutations in the serovars Newport, Virchow and Typhimurium and of Asp 87 mutations in the serovars Hadar and Kottbus. Seminati et al. (35) identified Asp $87 \rightarrow$ Tyr substitutions only in serovar Enteritidis (2 strains), Ser $83 \rightarrow$ Tyr only in serovar Anatum (3 strains), and Ser $83 \rightarrow$ Phe only in serovar Virchow (5 strains).

The occurrence of mutations in the Ser 83 codon is considered to be important for the development of FQ resistance (Piddock et al., 1998; Weigel et al., 1998; Weigel et al., 2002), a hypothesis that is supported by the results of this study. The Ser 83-mutant strains had a higher CipMIC (GMM $0.13 \mu \mathrm{g} / \mathrm{mL}$ ) than the Asp 87-mutant strains (GMM $0.061 \mu \mathrm{g} / \mathrm{mL}$ ). Similar results were described by Ling et al. (2003) and Giraud et al. (2006) that also showed an associa-

Table 1 - Distribution of the mutations observed in QRDR of the gyrA gene in Salmonella strains of different serovars with the corresponding minimal inhibitory concentration for ciprofloxacin (CipMIC) and geometric mean (GMM) data.

\begin{tabular}{|c|c|c|c|c|c|}
\hline Serovar & Mutation & N. strains & Strains origin & $\operatorname{CipMIC}^{\mathrm{a}}(\mu \mathrm{g} / \mathrm{mL})$ & $\mathrm{GMM}^{\mathrm{b}}(\mu \mathrm{g} / \mathrm{mL})$ \\
\hline \multirow[t]{11}{*}{ Enteritidis } & Ser $83 \rightarrow$ Tyr & 12 & Poultry (2); Epidemic (10) & 0.064 to 0.500 & 0.102 \\
\hline & Ser $83 \rightarrow$ Phe & 14 & Poultry (1); Epidemic (13) & 0.064 to 0.250 & 0.082 \\
\hline & Asp $87 \rightarrow$ Asn & 20 & Epidemic & 0.032 to 0.064 & 0.052 \\
\hline & Asp $87 \rightarrow$ Tyr & 4 & Epidemic & 0.032 to 0.064 & 0.058 \\
\hline & Asp $87 \rightarrow$ Gly & 2 & Epidemic & 0.032 & 0.032 \\
\hline & Ser $83 \rightarrow$ Thy/Asp $87 \rightarrow$ Gly & 1 & Epidemic & 0.125 & 0.125 \\
\hline & Ser $83 \rightarrow$ Thy/Asp $87 \rightarrow$ Asn & 3 & Poultry (1); Epidemic (2) & 0.125 to 0.250 & 0.220 \\
\hline & Ser $83 \rightarrow$ Phe/Asp $87 \rightarrow$ Asn & 4 & Poultry (1); Epidemic (3) & 0.064 to 0.125 & 0.105 \\
\hline & Ser $83 \rightarrow$ Phe/Asp $87 \rightarrow$ Gly & 1 & Epidemic & 0.125 & 0.125 \\
\hline & Ser $83 \rightarrow$ Phe/Asp $87 \rightarrow$ Tyr & 2 & Epidemic & 0.125 & 0.125 \\
\hline & Ala $131 \rightarrow$ Gly/Asp $87 \rightarrow$ Tyr & 1 & Epidemic & 0.125 & 0.125 \\
\hline \multirow[t]{2}{*}{ Typhimurium } & Ser $83 \rightarrow$ Tyr & 1 & Epidemic & 0.125 & 0.125 \\
\hline & Asp $87 \rightarrow$ Asn & 1 & Epidemic & 0.125 & 0.125 \\
\hline \multirow[t]{2}{*}{ Johannesburg } & Asp $87 \rightarrow$ Tyr & 2 & Epidemic & 0.064 & 0.064 \\
\hline & Ser $83 \rightarrow$ Tyr/Asp $87 \rightarrow$ Asn & 1 & Epidemic & 0.125 & 0.125 \\
\hline \multirow[t]{2}{*}{ Heidelberg } & Ser $83 \rightarrow$ Tyr & 1 & Poultry & 0.500 & 0.500 \\
\hline & Asp $87 \rightarrow$ Asn & 1 & Poultry & 0.064 & 0.064 \\
\hline Infantis & Ser $83 \rightarrow$ Phe & 1 & Epidemic & 0.064 & 0.064 \\
\hline Newport & Asp $87 \rightarrow$ Asn & 1 & Epidemic & 0.064 & 0.064 \\
\hline
\end{tabular}

${ }^{a}$ Souza et al., 2010; ${ }^{b}$ geometric mean. 
tion between the Ser 83 and Asp 87 mutations and the level of FQ resistance. The different substitutions in the same codon could also alter the binding of the quinolones to the DNA-gyrase complex.

Although the relationship between the mutations in the topoisomerase genes and quinolone resistance has been demonstrated, 39 of the NAL-resistant strains in the present study did not have mutations in the QRDRs of the four genes that were analyzed. Currently, it is known that the resistance to quinolones can also occur in the absence of mutations in gyrA. Nath and Maurya (2010) isolated ciprofloxacin-resistant Salmonella spp. strains without mutations in the QRDR of gyrA. Similarly, Gunell et al. (2009) observed a CipMIC of $0.5 \mu \mathrm{g} / \mathrm{mL}$ for a strain of $S$. Typhimurium without a mutation in the QRDR of gyrA.

The differences in antimicrobial susceptibility could be associated to additional mechanisms of resistance, such as efflux pumps over-expression and modification of porin regulation. Efflux pumps are transporter proteins that expel toxic substrates (including antibiotics) from inside cells to the outside. Efflux pumps over-expression can be caused by chromosomal mutations or in response to bacterial stress and could play an important role in resistance, especially when fluoroquinolones are involved (Eaves et al., 2004; Giraud et al., 2006; Piddock, 2002). Changes in permeability of the membrane with decreasing in intracellular penetration of the antimicrobial agent could also occur by a mechanism in which porins intervene (Hopkins et al., 2005).

In recent years, some research groups (Capoor et al., 2009; Crump et al., 2003; Friedman et al., 2001) have suggested that the QRDR of the gene gyrA could be amplified because mutations in the regions outside the QRDR domain that are associated with quinolone resistance had already been observed.

\section{PMQR}

In the present study, the qnrAl gene was detected in a strain of the serovar Enteritidis that was resistant to NAL with a CipMIC of $0.062 \mu \mathrm{g} / \mathrm{mL}$ without mutation in the QRDRs of the four genes analyzed (GenBank accession number GU731067). Similarly, Cui et al. (2009) found the $q n r$ genes $S 2, A$, and $B 6$ in Salmonella spp. that were susceptible to this fluoroquinolone. In contrast, Cavaco et al. (2007) found qnrA or $q n r S$ in Salmonella spp. strains that were resistant or had an intermediate sensitivity to NAL without mutations but showed a reduced susceptibility to Cip. Gay et al. (2006) also related the presence of $q n r$ genes, including $B 2, B 5, S 1$, and $S 2$, in Salmonella spp. strains with a reduced susceptibility to Cip.

The present study the gene qnrB19 was found in a strain of $S$. Corvallis from poultry origin with CipMIC of $0.5 \mu \mathrm{g} / \mathrm{mL}$ and without mutations in the QRDRs of the four genes that were analyzed (GenBank accession number GU731069). This gene was also identified in strains of $S$.
Typhimurium with a reduced susceptibility to Cip in France (Cattoir et al., 2007) and in the Netherlands (GarciaFernandez et al., 2009).

The qnr gene, always associated with plasmids genes of quinolones resistance in Enterobacteriaceae (GarciaFernandez et al., 2009; Hopkins et al., 2005; Jacoby et al., 2003), has already been found in Salmonella strains with different levels of susceptibility to Cip. However, in this work the presence of plasmids in the strains was not evaluated. According to previous studies (Castanheira et al., 2007; Cavaco and Aarestrup, 2009; Jacoby et al., 2003; Li, 2005; Minarini et al., 2008; Zhao et al., 2008) the presence of this gene confers a low level of resistance to Cip while also facilitating the development of mutations in the QRDR of the gyrA gene. Chong et al. (2010) and Jacoby et al. (2003) reported that the increase in FQ resistance resulting from the presence of $q n r$ genes could reduce the clinical effectiveness of this class of antibiotics. However, according Jacoby et al. (2009), the precise level of the involvement of plasmid genes in the resistance to FQs is still poorly understood when compared with our understanding of other mechanisms of resistance.

In conclusion, despite previous detection of qnrAl, $q n r B 2$, qnrB8, qnrVC1, qnrVC2 genes in other bacterial species in Brazil (28), it is the first report of the $q n r$ gene in Salmonella, and also the first detection of the qnrB19 gene in this country. The results suggest that an integrated approach between the medical and veterinary communities is required to effectively control drug resistance. It was also shown that the sensitivity to NAL seems to be a good phenotypic indication to differentiate between strains with and without a mutation in the QRDR, however the double mutation in gyrA did not cause resistance to ciprofloxacin. The mechanisms by which enteric zoonotic bacteria become resistant need to be continuously monitored in order to prevent the selection of strains with a reduced susceptibility and resistance to quinolones. Only through these measures may correct preventive decisions be taken to continue the effective therapeutic use of this class of antibiotics.

\section{Acknowledgments}

The authors would like to thank CNPq and Capes for financial support. Thanks are also to Dra Lina Cavaco for providing the qnrA, qnrB1, qnrB5, qnrS1, qnrC, qnrD and $a a c(6) I b-c r$ strains used as positive controls. Thanks to Marcelo Moreschi for the writing suggestions.

\section{References}

Aarestrup FM, Wiuff C, Molbak K, Threlfall EJ (2003) Is it time to change fluoroquinolone breakpoints for Salmonella spp.? Antimicrob Agents Chemother 47:827-829.

Arkin R (2008) posting date. Escherichia coli O157:H7 Foodborne Illness and the Usefulness of the Critical Path in FDA's Work to Combat It. FDA Veterinarian Newsletter. 
23:1.http:/www.fda.gov/AnimalVeterinary/NewsEvents/F DA Veterinarian Newsletter/ucm083980.htm.

Capoor MR, Nair D, Walia NS, Routela RS, Grover SS, Deb M, Aggarwal P, Pillai PK, Bifani PJ (2009) Molecular analysis of high-level ciprofloxacin resistance in Salmonella enterica serovar Typhi and $S$. Paratyphi A: Need to expand the QRDR region? Epidemiol Infect 137:871-878.

Castanheira M, Pereira AS, Nicoletti AG, Pignatari AC, Barth AL, Gales AC (2007) First report of plasmid-mediated qnrA1 in a ciprofloxacin-resistant Escherichia coli strain in Latin America. Antimicrob Agents Chemother 51:15271529.

Cattoir V, Weill FX, Poirel L, Fabre L, Soussy CJ, Nordmann P (2007) Prevalence of qnr genes in Salmonella in France. J Antimicrob Chemother 59:751-754.

Cavaco LM, Hendriksen RS, Aarestrup FM (2007) Plasmid-mediated quinolone resistance determinant qnrS1 detected in Salmonella enterica serovar Corvallis strains isolated in Denmark and Thailand. J Antimicrob Chemother 60:704706.

Cavaco LM, Aarestrup FM (2009) Evaluation of quinolones for use in detection of determinants of acquired quinolone resistance, including the new transmissible resistance mechanisms qnrA, qnrB, qnrS, and aac(6')ib-cr, in Escherichia coli and Salmonella enterica and determinations of wildtype distributions. J Clin Microbiol 47:2751-2758.

Chong YP, Choi SH, Kim ES, Song EH, Lee EJ, Park KH, Cho $\mathrm{OH}, \mathrm{Kim} \mathrm{SH}$, Lee SO (2010) Bloodstream infections caused by qnr-positive Enterobacteriaceae: Clinical and microbiologic characteristics and outcomes. Diagn Microbiol Infect Dis 67:70-77.

Clinical and Laboratory Standards Institute (CLSI). (2011). Performance Standards for Antimicrobial Susceptibility Testing. Twenty-first Informational Supplement, Document M100-S21, Wayne PA.

Crump JA, Barrett TJ, Nelson JT, Angulo FJ (2003) Reevaluating fluoroquinolone breakpoints for Salmonella enterica serotype Typhi and for non-Typhi salmonellae. Clin Infect Dis 37:75-81

Cui S, Li J, Sun Z, Hu C, Jin S, Li F, Guo Y, Ran L, Ma Y (2009) Characterization of Salmonella enterica isolates from infants and toddlers in Wuhan, China. J Antimicrob Chemother 63:87-94.

Eaves DJ, Ricci V, Piddock LJ (2004) Expression of $a c r B$, $a c r F$, acrD, marA, and soxS in Salmonella enterica serovar Typhimurium: Role in multiple antibiotic resistance. Antimicrob Agents Chemother 48:1145-1150.

Ercis S, Erdem B, Hascelik G, Gur D (2006) Nalidixic acid resistance in Salmonella strains with decreased susceptibility to ciprofloxacin isolated from humans in Turkey. Jpn J Infect Dis 59:117-119.

Fonseca EL, dos Santos Freitas F, Vieira VV, Vicente AC (2008) New $q n r$ gene cassettes associated with superintegron repeats in Vibrio cholerae O1. Emerg Infect Dis 14:11291131.

Friedman SM, Lu T, Drlica K (2001) Mutation in the DNA gyrase a gene of Escherichia coli that expands the quinolone resistance-determining region. Antimicrob Agents Chemother 45:2378-2380.

Garcia-Fernandez A, Fortini D, Veldman K, Mevius D, Carattoli A (2009) Characterization of plasmids harbouring qnrS1,
qnrB2 and qnrB19 genes in Salmonella. J Antimicrob Chemother 63:274-281.

Gay K, Robicsek A, Strahilevitz J, Park CH, Jacoby G, Barrett TJ, Medalla F, Chiller TM, Hooper DC (2006) Plasmid-mediated quinolone resistance in non-Typhi serotypes of Salmonella enterica. Clin Infect Dis 43:297-304.

Giraud E, Baucheron S, Cloeckaert A (2006) Resistance to fluoroquinolones in Salmonella: Emerging mechanisms and resistance prevention strategies. Microbes Infect 8:1937-1944.

Giraud E, Brisabois A, Martel JL, Chaslus-Dancla E (1999) Comparative studies of mutations in animal isolates and experimental in vitro- and in vivo-selected mutants of Salmonella spp. Suggest a counter selection of highly fluoroquinolone-resistant strains in the field. Antimicrob Agents Chemother 43:2131-2137.

Gunell M, Webber MA, Kotilainen P, Lilly AJ, Caddick JM, Jalava J, Huovinen P, Siitonen A, Hakanen AJ (2009) Mechanisms of resistance in nontyphoidal Salmonella enterica strains exhibiting a nonclassical quinolone resistance phenotype. Antimicrob Agents Chemother 53:3832-3836.

Hopkins KL, Davies RH, Threlfall EJ (2005) Mechanisms of quinolone resistance in Escherichia coli and Salmonella: Recent developments. Int J Antimicrob Agents 25:358-373.

Jacoby GA, Gacharna N, Black TA, Miller GH, Hooper DC (2009) Temporal appearance of plasmid-mediated quinolone resistance genes. Antimicrob Agents Chemother 53:1665-1666.

Jacoby GA, Chow N, Waites KB (2003) Prevalence of plasmidmediated quinolone resistance. Antimicrob Agents Chemother 47:559-562.

Kownhar H, Shankar EM, Rajan R, Rao UA (2007) Emergence of nalidixic acid-resistant Salmonella enterica serovar Typhi resistant to ciprofloxacin in India. J Med Microbiol 56:136137.

Li XZ (2005) Quinolone resistance in bacteria: Emphasis on plasmid-mediated mechanisms. Int $\mathrm{J}$ Antimicrob Agents 25:453-463.

Ling JM, Chan EW, Lam AW, Cheng AF (2003) Mutations in topoisomerase genes of fluoroquinolone-resistant Salmonellae in Hong Kong. Antimicrob Agents Chemother 47:3567-3573.

Martinez-Martinez L, Garcia I, Ballesta S, Benedi VJ, Hernandez-Alles S, Pascual A (1998) Energy-dependent accumulation of fluoroquinolones in quinolone-resistant Klebsiella pneumoniae strains. Antimicrob Agents Chemother 42:1850-1852.

Minarini LA, Poirel L, Cattoir V, Darini AL, Nordmann P (2008) Plasmid-mediated quinolone resistance determinants among enterobacterial isolates from outpatients in Brazil. J Antimicrob Chemother 62:474-478.

Nath G, Maurya P (2010) Drug resistance patterns in Salmonella enterica subspecies enterica serotype Typhi strains isolated over a period of two decades, with special reference to ciprofloxacin and ceftriaxone. Int $\mathrm{J}$ Antimicrob Agents $35: 482-485$

Piddock LJ, Ricci V, McLaren I, Griggs DJ (1998) Role of mutation in the gyrA and parC genes of nalidixic-acid-resistant Salmonella serotypes isolated from animals in the United Kingdom. J Antimicrob Chemother 41:635-641. 
Piddock LJ (2002) Fluoroquinolone resistance in Salmonella serovars isolated from humans and food animals. FEMS Microbiol Rev 26:3-16.

Ricci V, Piddock LJ (2009) Ciprofloxacin selects for multidrug resistance in Salmonella enterica serovar Typhimurium mediated by at least two different pathways. J Antimicrob Chemother 63:909-916.

Robicsek A, Jacoby GA, Hooper DC (2006) The worldwide emergence of plasmid-mediated quinolone resistance. Lancet Infect Dis 6:629-640.

Robicsek A, Strahilevitz J, Sahm DF, Jacoby GA, Hooper DC (2006) Qnr prevalence in ceftazidime-resistant Enterobacteriaceae isolates from the United States. Antimicrob Agents Chemother 50:2872-2874.

Seminati C, Mejia W, Mateu E, Martin M (2005) Mutations in the quinolone-resistance determining region (QRDR) of Salmonella strains isolated from pigs in Spain. Vet Microbiol 106:297-301.

Sjolund-Karlsson M, Howie R, Rickert R, Krueger A, Thuy T, Tran T, Zhao S, Ball T, Haro J (2010) Plasmid-mediated quinolone resistance among non-typhi Salmonella enterica isolates, USA. Emerg Infect Dis 16:1789-1791.

Soto SM, Gonzalez-Hevia MA, Mendoza MC (2003) Antimicrobial resistance in clinical isolates of Salmonella enterica serotype Enteritidis: Relationships between mutations conferring quinolone resistance, integrons, plasmids and genetic types. J Antimicrob Chemother 51:1287-1291.

Souza RB, Ferrari RG, Magnani M, Kottwitz LBM, Alcocer I, Tognim MCB, Oliveira TCRM (2010) Ciprofloxacin sus- ceptibility reduction of Salmonella strains isolated from outbreaks. Braz J Microbiol 41:497-500.

Tran JH, Jacoby GA, Hooper DC (2005) Interaction of the plasmid-encoded quinolone resistance protein qnrA with Escherichia coli topoisomerase IV. Antimicrob Agents Chemother 49:3050-3052.

Vashist J, Kapoor R, Kapil A, Yennamalli R, Subbarao N, Rajeswari MR (2009) Interaction of nalidixic acid and ciprofloxacin with wild type and mutated quinolone-resistancedetermining region of DNA gyrase. Indian $\mathrm{J}$ Biochem Biophys 46:147-153.

Wang M, Guo Q, Xu X, Wang X, Ye X, Wu S, Hooper DC (2009) New plasmid-mediated quinolone resistance gene, qnrC, found in a clinical isolate of Proteus mirabilis. Antimicrob Agents Chemother 53:1892-1897.

Weigel LM, Anderson GJ, Tenover FC (2002) DNA-gyrase and topoisomerase IV mutations associated with fluoroquinolone resistance in Proteus mirabilis. Antimicrob Agents Chemother 46:2582-2587.

Weigel LM, Steward CD, Tenover FC (1998) gyrA mutations associated with fluoroquinolone resistance in eight species of Enterobacteriaceae. Antimicrob Agents Chemother 42:2661-2667.

Zhao X, Xu X, Zhu D, Ye X, Wang M (2010) Decreased quinolone susceptibility in high percentage of Enterobacter cloacae clinical isolates caused only by $q n r$ determinants. Diagn Microbiol Infect Dis 67:110-113.

All the content of the journal, except where otherwise noted, is licensed under a Creative Commons License CC BY-NC. 